\title{
CORRIGENDUM
}

\section{The rat tyrosine phosphatase $\eta$ increases cell adhesion by activating c-Src through dephosphorylation of its inhibitory phosphotyrosine residue}

I Le Pera, R Iuliano, T Florio, C Susini, F Trapasso, M Santoro, L Chiariotti, G Schettini, G Viglietto and A Fusco

Oncogene (2016) 35, 5456; doi:10.1038/onc.2016.138; published online 18 July 2016

Correction to: Oncogene (2005) 24, 3187-3195; doi:10.1038/sj. onc.1208510; published online 21 February 2005

The authors wish to make corrections to Figures 3 and $5 a$ of the original publication.

The authors wish to notify the readers that an incorrect panel was submitted for Figure 3. The corrected figure is included here.

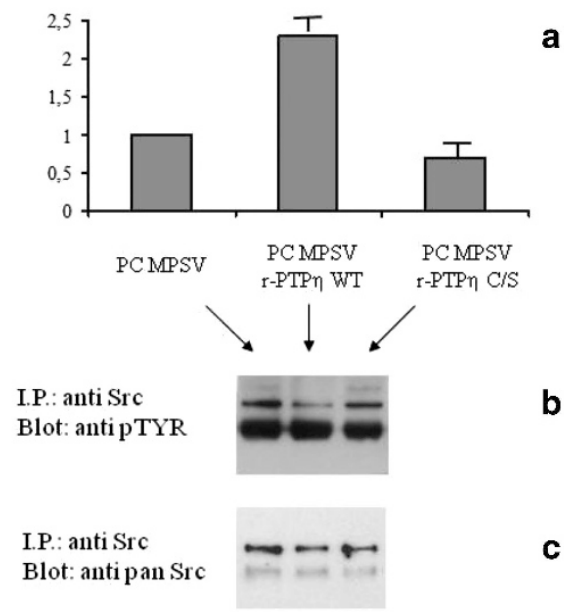

The figure here shows the raw data of the correct panel, which confirms the results reported in the paper, for example, that $r$-PTPh expression in PC-MPSV cells reduces overall phosphotyrosine Src content with respect to parental cells, as well as to cells transfected with the inactive r-PTPh C/S mutant.

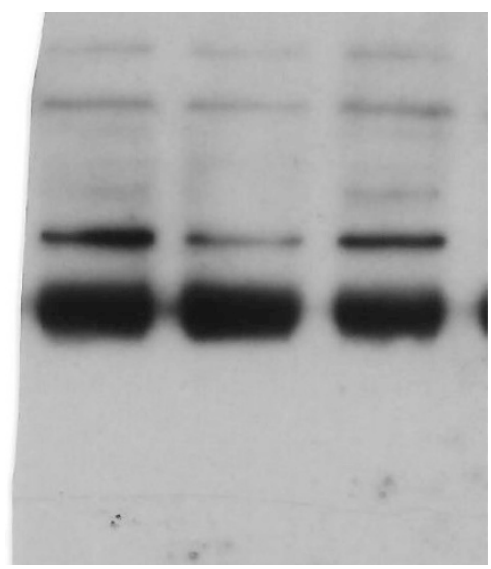

The authors also wish to notify the readers that the orientation of the samples were wrongly indicated in Figure 5a. The figure here shows the correct orientation from left to right: PC-MPSV/r-PTPh C/S, PC-MPSV/r-PTPh and PC-MPSV, respectively, rather than as wrongly indicated in the original manuscript: PC-MPSV, PC-MPSV/ r-PTPh and PC-MPSV/r-PTPh C/S, respectively. There is no change in the meaning of the results as the intensity of the three bands that were shown in the panel for normalization is very similar.

a

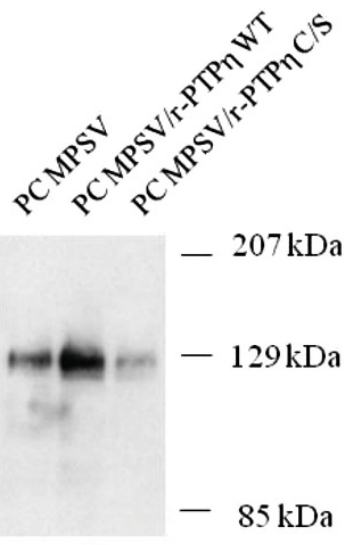

I.P.: anti FAK

Blot: anti FAK

Figure 5. 